\title{
Will Comprehensive Assessment from Esophagus to Large Bowel Revive the Momentum for Radionuclide Gastrointestinal Transit Studies?
}

\author{
Federica Guidoccio, Italia Paglianiti, Giuseppe Boni, and Giuliano Mariani \\ Regional Center of Nuclear Medicine, University of Pisa and University Hospital of Pisa, Pisa, Italy
}

$\mathbf{I}_{\mathrm{n}}$ this issue of The Journal of Nuclear Medicine, Antoniou et al. describe a novel scintigraphic procedure designed to evaluate in a physiologic manner the transit of both the liquid and the solid components of a meal through virtually the entire gastrointestinal tract, starting with esophageal transit (1). Although the procedure is somewhat complex and laborious, it does offer the advantage of combining assessment of esophageal, gastric, small-bowel, and colonic transit so as to obtain quantitative information on either the overall transit time or the separate components of transit from the esophagus to the more distal end of the gastrointestinal tract; the procedure also entails separate assessments of the liquid and solid components of a meal.

This scenario has always attracted the attention of nuclear physicians, and indeed the first radionuclide technique combining evaluation of gastric emptying with evaluation of small-bowel and colon transit was published in 1975 (2). Various approaches have subsequently been described, and reference ranges for healthy subjects have been defined $(3,4)$; nevertheless, to date there is

\section{See page 721}

no scintigraphic protocol univocally recognized as the standard adopted worldwide, even for separate assessment of transit in the different portions of the gastrointestinal tract, that is, esophagus, stomach, small bowel, and large bowel-although the recent publication of guidelines jointly developed by the Society of Nuclear Medicine and Molecular Imaging (SNMMI) and the European Association of Nuclear Medicine (EANM) constitutes an important move in this direction (5).

The study by Antoniou et al. is different from others for various reasons, the most obvious being combination in a single examination of so many components of gastrointestinal pathophysiology, both regionwise (esophagus to large bowel) and mealwise (solid and liquid). One current drawback of the study, that is, the development of reference ranges for the various functional

Received Feb. 18, 2015; revision accepted Feb. 25, 2015.

For correspondence contact: Giuliano Mariani, Regional Center of Nuclear Medicine, University of Pisa, Via Roma 67, I-56126 Pisa, Italy.

E-mail: giuliano.mariani@med.unipi.it

Published online Mar. 12, 2015.

COPYRIGHT (C) 2015 by the Society of Nuclear Medicine and Molecular Imaging, Inc.

DOI: $10.2967 /$ jnumed.115.154963 parameters based on a relatively small population of healthy subjects $(n=18)$, will certainly be overcome as experience from the same group or from other groups stimulated by this proposal will grow. Another crucial issue addressed by Antoniou et al. is that their protocol for comprehensive assessment of transit from esophagus to large bowel is designed to meet the clinical demand that cannot be adequately met by separate assessment of transit for single portions of the gastrointestinal tract (esophagus, stomach, small bowel, and large bowel)—most likely one of the reasons why such radionuclide transit studies have never really grown to represent the definite solution for patients with dyspeptic syndromes.

It is important to consider that dyspeptic symptoms are common in patients with either gastric or small-bowel dysmotility and constitute a common complaint in patients with esophageal dysmotility as well. Moreover, although constipation or diarrhea is the predominant symptom directing attention to some abnormality in large-bowel transit, patients with these symptoms often have additional complaints that overlap those more typical of the upper gastrointestinal tract (i.e., dysphagia or dyspepsia). In fact, it is now well known that transit abnormalities can concomitantly affect more than one gastrointestinal region (6), and the assumption that dyspepsia means motility disorder of the upper gastrointestinal tract whereas diarrhea and constipation mean disorder of the lower gastrointestinal tract is not systematically true. Thus, by adopting a scintigraphic protocol enabling routine investigation for small- and large-bowel dysmotility in addition to esophageal transit and gastric emptying, we might be able to characterize (and therefore to correctly diagnose) gastrointestinal dysmotility syndromes in more patients than we can with separate (often incomplete) assessments of transit through the various regions of the gastrointestinal tract.

On the other hand, it should be considered that, in the face of some relative inadequacy in radionuclide transit studies when performed separately and incompletely, nonradionuclide procedures that gastroenterologists can use in their own environments, such as various breath tests, ultrasound, and more recently MR and wireless motility capsules, portend to yield the same diagnostic information as that derived from classic radionuclide gastrointestinal transit studies. Nevertheless, it is also known that correspondence between nonradionuclide- and radionuclide-based modalities is less than optimal, as radionuclide procedures reflect as closely as possible the true physiology of gastrointestinal motility (7-11). Thus, variable degrees of interaction among different specialists (gastroenterologists vis-à-vis nuclear physicians) make it difficult to standardize protocols among different clinical centers, as they favor local variations that are introduced to meet specific expectations of the referring specialists. In 
addition to having variations in clinical protocols, radionuclide gastrointestinal transit studies still suffer from a considerable heterogeneity among different nuclear medicine centers, although such heterogeneity is expected to decrease after publication of the joint SNMMI/ EANM guidelines (5). These differences are related to important practical aspects not only of the procedure itself (such as the preparation and composition of the radiolabeled meal, acquisition modalities, and quantitative/semiquantitative assessment of the data obtained) but also of the way the results are interpreted and presented. Furthermore, the standardized basic components of a radiolabeled meal are also variably available in different countries, such as commercially prepared egg white, which is available only in the United States. Other differences derive from country-specific variations in the balance of the basic nutrients (carbohydrates, proteins, fats) and in variable palatability and composition of food in different ethnic cultures. All these factors probably explain why, from the nuclear medicine point of view, gastrointestinal transit studies have generally remained a niche activity with respect to the imaging procedures most commonly performed in any nuclear medicine center. Moreover, insufficient attention is paid to the fact that a properly performed radionuclide gastrointestinal transit study is extremely useful not only in the diagnostic approach to a patient with a gastrointestinal motility disorder but also in assessing the efficacy of therapy; in this regard, it should be kept in mind that most drugs used clinically to treat a motility disorder affecting, for example, gastric emptying actually also interfere with the motility of other portions of the gastrointestinal tract.

Is it always necessary to perform a comprehensive test for gastrointestinal transit and motility, including solid and liquid phases? Experience is growing that investigating the motility of the entire gastrointestinal tract can reduce diagnostic errors or incomplete diagnoses, leading in several instances to modified clinical management and therapy. For instance, gastroparesis can go undetected in $20 \%-40 \%$ of the cases (12), whereas its detection can increase by about one third when the acquisition time is extended from 2 to $4 \mathrm{~h}$ $(13,14)$; furthermore, assessing liquid gastric emptying improves the detection of gastroparesis by another third $(15,16)$.

Why do we need to standardize the comprehensive radionuclide gastrointestinal transit study? Even if the joint SNMMI/EANM guidelines constitute an important milestone in the process of standardization, they concern only small-bowel and large-bowel transit (5); further multidisciplinary interaction is necessary to develop similar guidelines, and therefore standardization, for the upper gastrointestinal tract, that is, esophageal transit and gastric emptying. Continuing discussion between the gastroenterologist and the nuclear physician on specific clinical cases encountered in the daily routine will obviously result in an improved possibility of achieving a clear, complete, and definite diagnosis. The diagnosis will be clear because the nuclear physician will know the diagnostic dilemma of the gastroenterologist, and the diagnosis will be complete and definite because the nuclear physician will provide a comprehensive evaluation of the various portions of the entire gastrointestinal tract. In this scenario, certainly standardization plays an important role in promoting better interaction between nuclear physicians and gastroenterologists across different clinical centers.

How can the multidisciplinary approach improve the evaluation of patients with gastrointestinal motility disorders? The multidisciplinary approach is important to patients with motility disorders of the gastrointestinal tract and should include a team of health care experts such as gastroenterologists, nutritionists, radiologists, and nuclear physicians. Each of these experts will contribute to some aspects of the characterization-and therefore to correct diagnosis and clinical management-of patients with gastrointestinal motility disorders. Such a synergistic vision of the patient is superior to the simple arithmetic sum of each contribution.

In conclusion, on the basis of the promise offered by comprehensive radionuclide evaluation of the gastrointestinal transit, we envision a future where the clinical feedback on a patient-to-patient basis will guarantee that the information provided by such a protocol is indeed of value to the specialist in gastroenterology and ultimately to the patient. Thus, we encourage the nuclear medicine and the gastroenterology communities to continue to work synergistically toward developing joint guidelines that include both the upper and the lower gastrointestinal tracts, in the avenue opened by the work of Antoniou et al. and with the goal of treating all motility disorders of the gastrointestinal tract as pertaining to a single organ rather than to separate portions.

\section{DISCLOSURE}

No potential conflict of interest relevant to this article was reported.

\section{REFERENCES}

1. Antoniou AJ, Raja S, El-Khouli R, et al. Comprehensive radionuclide esophagogastrointestinal transit study: methodology, reference values, and initial clinical experience. J Nucl Med. 2015;56:721-727.

2. Waller SL. Differential measurement of small and large bowel transit times in constipation and diarrhea: a new approach. Gut. 1975;16:372-378.

3. Read NW, Al-Janabi MN, Holgate AM, et al. Simultaneous measurement of gastric emptying, small bowel residence and colonic filling of a solid meal by the use of the gamma camera. Gut. 1986;27:300-308.

4. Madsen JL, Jensen M. Gastrointestinal transit of technetium-99m-labeled cellulose fiber and indium-111-labeled plastic particles. J Nucl Med. 1989;30: 402-406.

5. Maurer AH, Camilleri M, Donohoe K, et al. The SNMMI and EANM practice guideline for small-bowel and colon transit 1.0. J Nucl Med. 2013;54: 2004-2013.

6. Rao SSC, Camilleri M, Hasler WL, et al. Evaluation of gastrointestinal transit in clinical practice: position paper of the American and European Neurogastroenterology and Motility Societies. Neurogastroenterol Motil. 2011;23:8-23.

7. Madsen JL, Larsen NE, Hilsted J, Worning H. Scintigraphic determination of gastrointestinal transit times: a comparison with breath hydrogen and radiologic methods. Scand J Gastroenterol. 1991;26:1263-1271.

8. Sciarretta G, Furno A, Mazzoni M, Garagnani B, Malaguti P. Lactulose hydrogen breath test in orocecal transit assessment: critical evaluation by means of scintigraphic method. Dig Dis Sci. 1994;39:1505-1510.

9. Miller MA, Parkman HP, Urbain JL, et al. Comparison of scintigraphy and lactulose breath hydrogen test for assessment of orocecal transit: lactulose accelerates small bowel transit. Dig Dis Sci. 1997;42:10-18.

10. Kuo B, McCallum RW, Koch KL, et al. Comparison of gastric emptying of a nondigestible capsule to a radio-labelled meal in healthy and gastroparetic subjects. Aliment Pharmacol Ther. 2008;27:186-196.

11. Boillat CS, Gaschen FP, Gaschen L, Stout RW, Hosgood GL. Variability associated with repeated measurements of gastrointestinal tract motility in dogs obtained by use of a wireless motility capsule system and scintigraphy. Am J Vet Res. 2010;71:903-908.

12. Tack J, Bisschops RAF, Sarnelli G. Pathophysiology and treatment of functional dyspepsia. Gastroenterology. 2004;127:1239-1255.

13. Guo J-P, Maurer AH, Fisher RS, Parkman HP. Extending gastric emptying scintigraphy from two to four hours detects more patients with gastroparesis. Dig Dis Sci. 2001;46:24-29.

14. Ziessman HA, Goetze S, Bonta D, Ravich W. Experience with a new standardized 4-hr gastric emptying protocol. J Nucl Med. 2007;48:568-572.

15. Ziessman HA, Chander A, Ramos A, Wahl RL, Clark JO. The added value of liquid gastric emptying compared to solid emptying alone. J Nucl Med. 2009;50:726-731.

16. Sachdeva P, Malhotra N, Pathikonda M, et al. Gastric emptying of solids and liquids for evaluation for gastroparesis. Dig Dis Sci. 2011;56:1138-1146. 